\title{
Techno-Economical feasibility of bio-cellulose membrane along with polyethylene film as a separator for lead-acid batteries
}

\author{
Sung-Hee Roh, ${ }^{\dagger, \#}$ Gowthami Palanisamy, ${ }^{\dagger, \# ~ T . ~ S a d h a s i v a m, ~}{ }^{\star, \S}$ Jae-Eun Jin,, Jin-Yong \\ Shim, " and Ho-Young Jung*,,,,$\perp$ \\ ${ }^{\dagger}$ College of General Education, Chosun University, \\ 309 Pilmoon-daero, Dong-gu, Gwangju 61452, Republic of Korea \\ $\$$ Department of Environment \& Energy Engineering, Chonnam National University, \\ 77 Yongbong-ro, Buk-gu, Gwangju 61186, Republic of Korea \\ ${ }^{\S}$ Center for Energy Storage System, Chonnam National University, \\ 77 Yongbong-ro, Buk-gu, Gwangju 61186, Republic of Korea \\ ŁEnergy Planet Co. Ltd., 308, Venture Center, Techno Park, 333 Cheomdangwagi-ro, Buk-gu, \\ Gwangju 61186, Republic of Korea

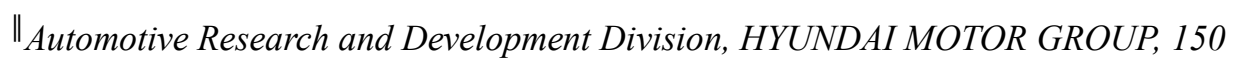 \\ Hyundaiyeonguso-ro, Namyang-eup, Hwaseong-si, Gyeonggi-do, 445-706, Republic of Korea.
}

\#These two authors (Sung-Hee Roh and Gowthami Palanisamy) contributed equally to this work and considered as a co-first author.

*Author to whom correspondence should be addressed.

Corresponding author details

Professor: Ho-Young Jung Email:jungho@jnu.ac.kr; jungho@chonnam.ac.kr

Tel. : +82-62-530-1865

Fax: +82-62-530-1859

6 pages (including title page), 5 Figures

Figure S1. BET curve of (a) AGM separator, (b) BC membrane and (c) PE separator.

Figure S2. Discharge capacity of AGM battery and BC-PE battery system.

Figure S3. Long-term cycle test performances of BC-PE battery at $0.1 \mathrm{~A}$ current.

Figure S4. Cycle performance of BC-PE battery at $0.1 \mathrm{~A}$ after (a) $100^{\text {th }}$, (b) 1000 , (c) 5000, and (d) 10000 cycles.

Figure S5. Cycle performance of BC-PE battery at $0.1 \mathrm{~A}$ after (e) 15000 and (f) 20000 cycles. 

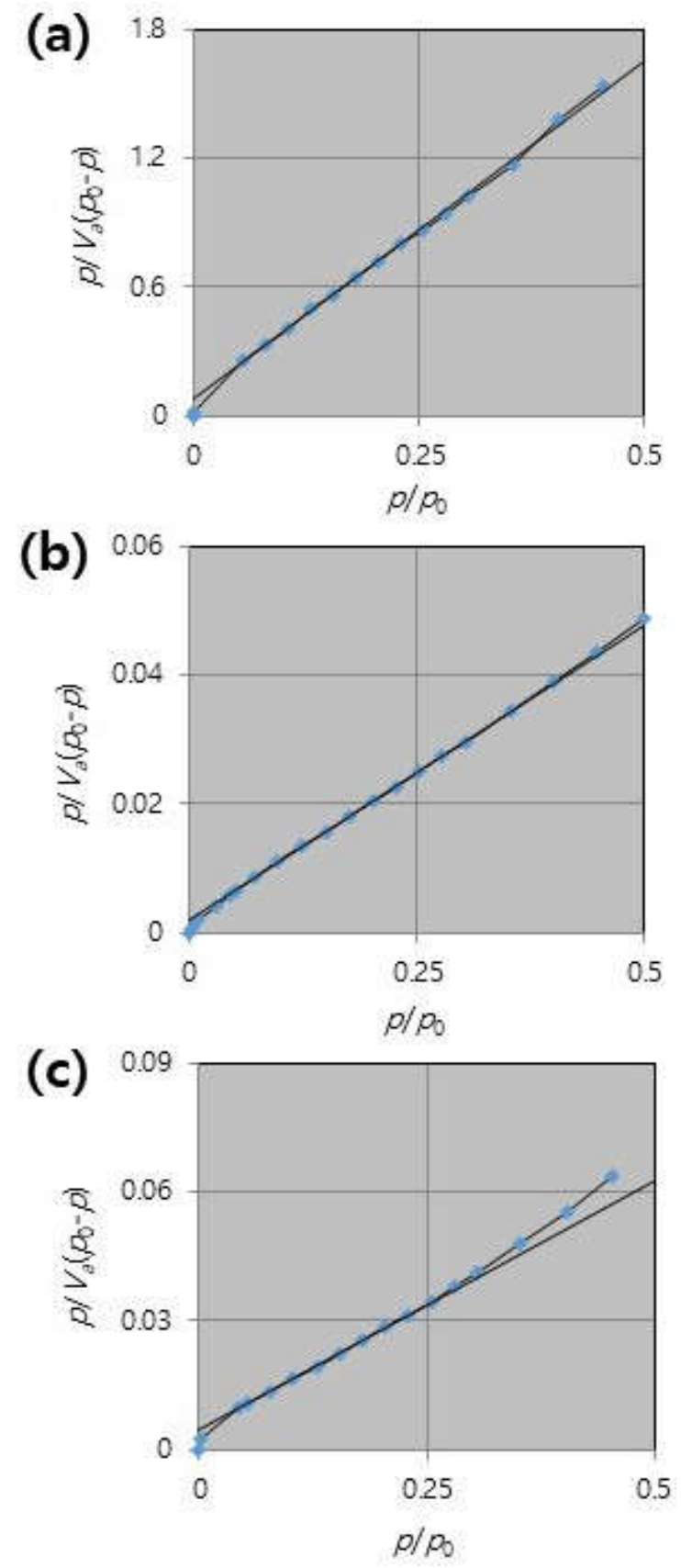

Figure S1. BET curve of (a) AGM separator, (b) BC membrane and (c) PE separator. 


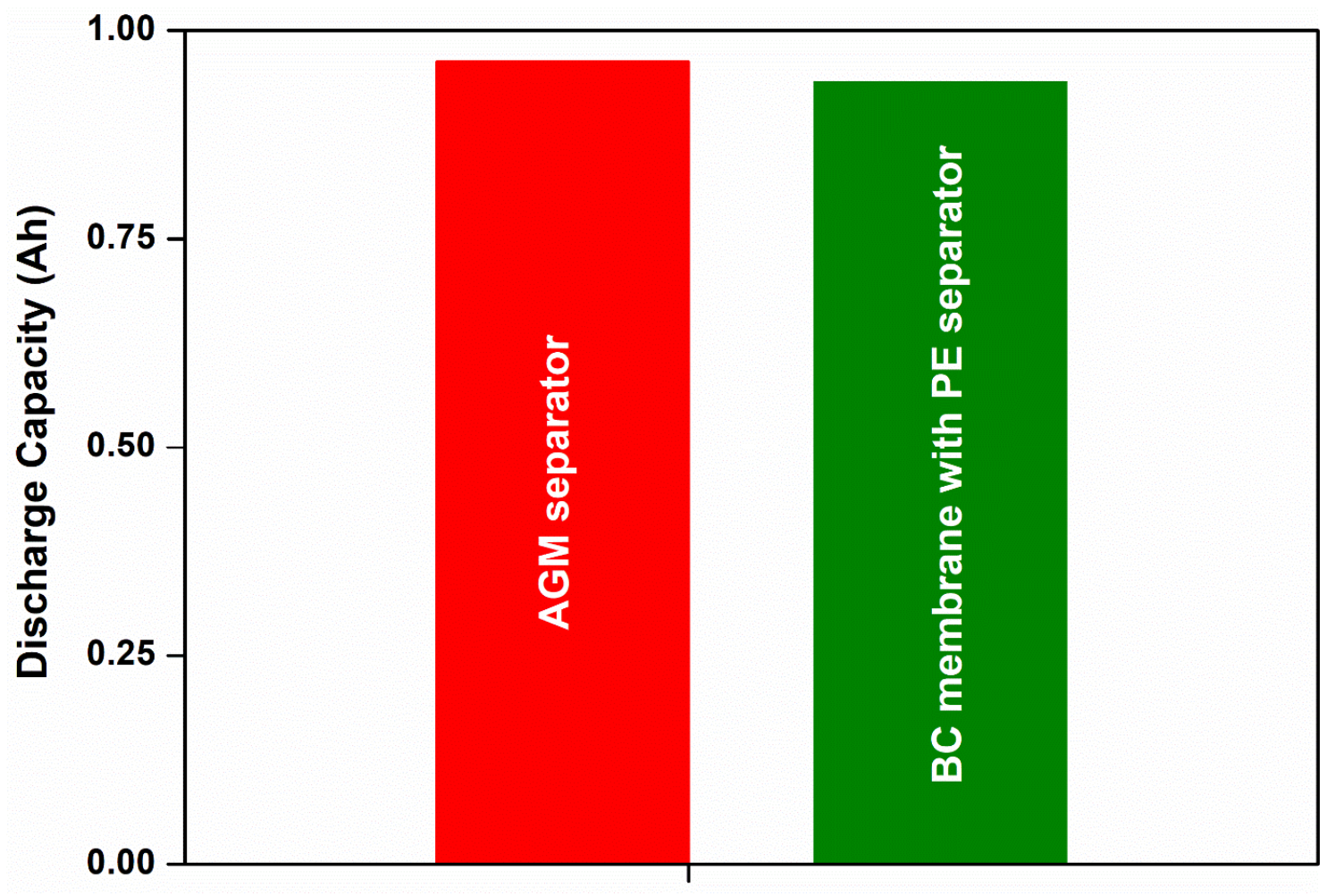

Figure S2. Discharge capacity of AGM battery and BC-PE battery system. 


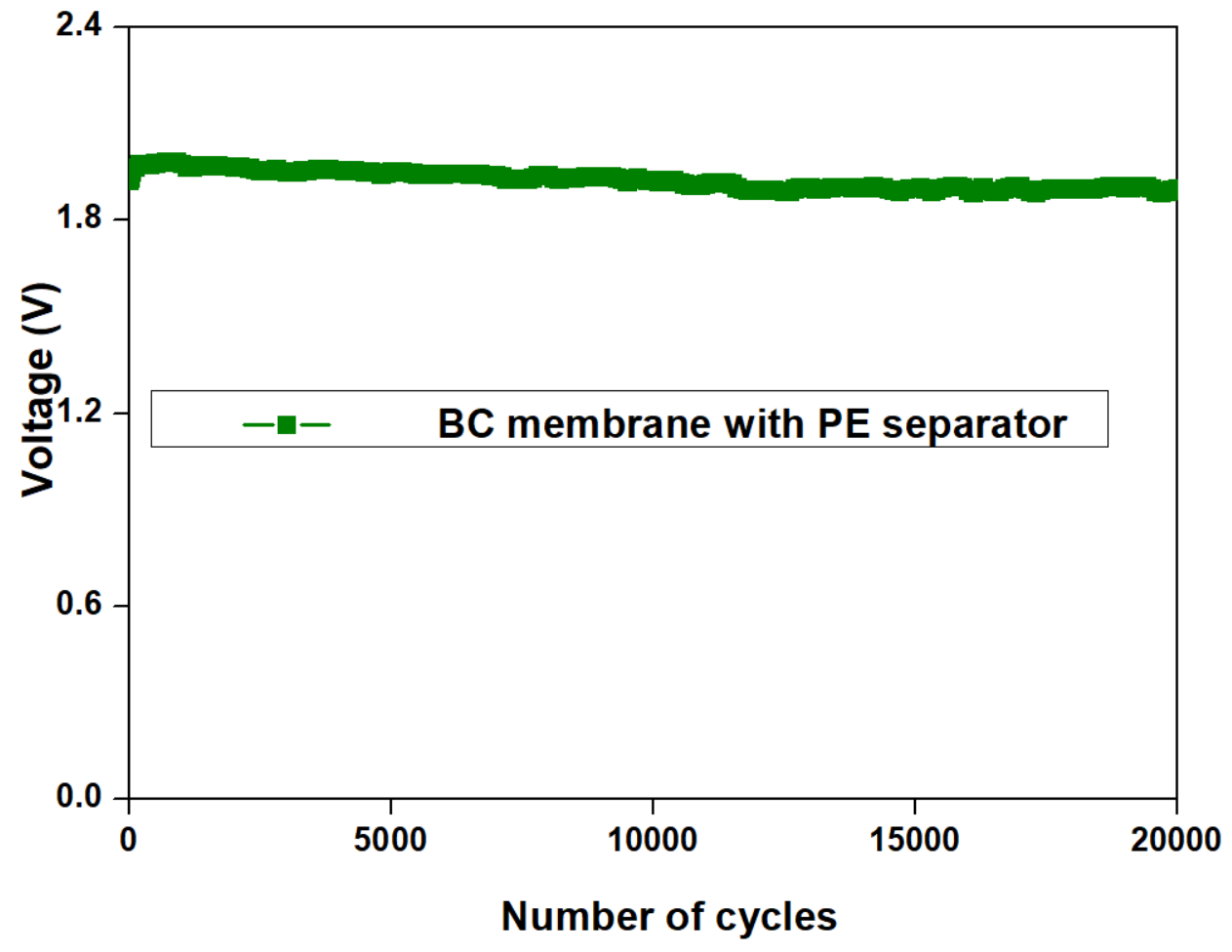

Figure S3. Long-term cycle test performances of BC-PE battery at 0.1A current. 

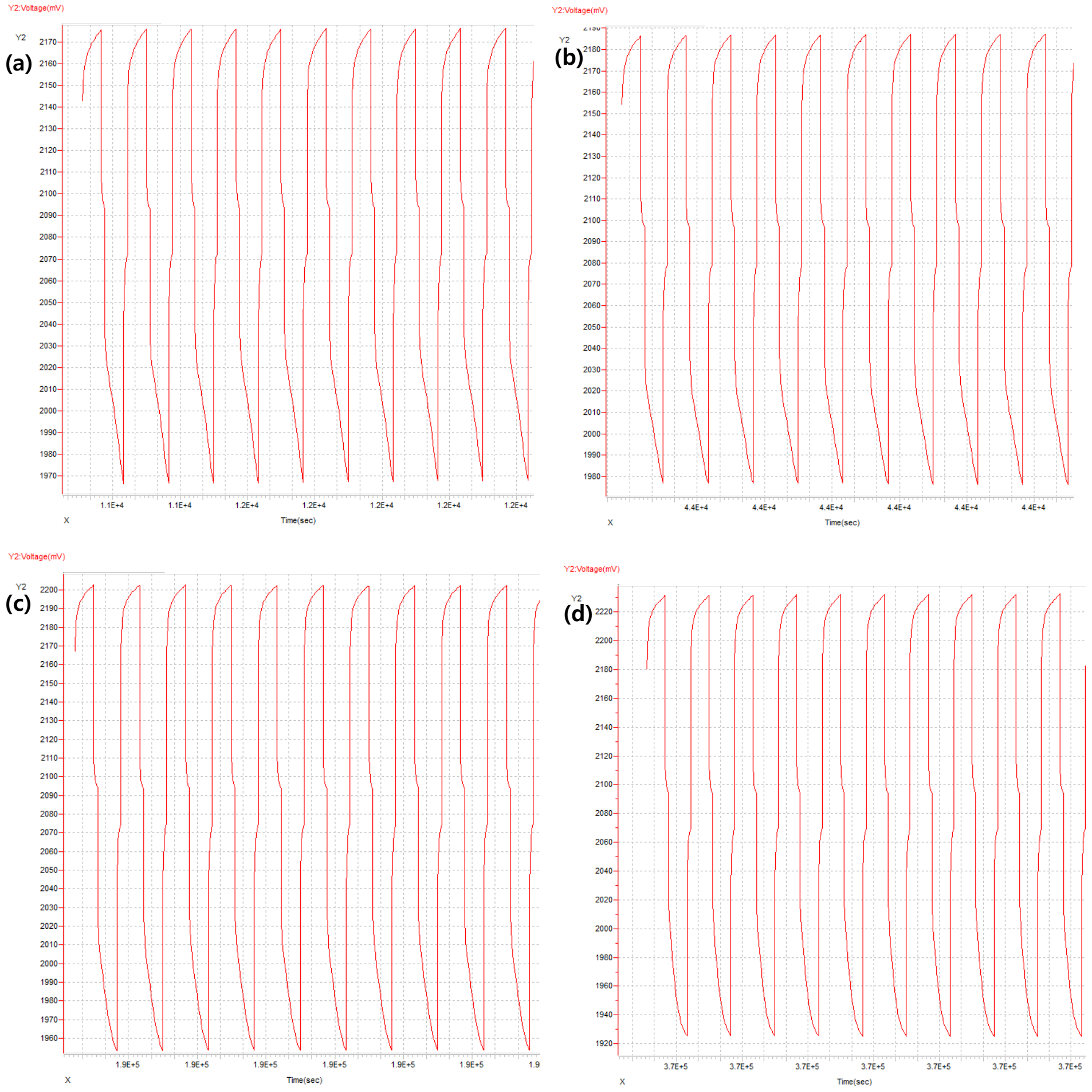

Figure S4. Cycle performance of BC-PE battery at 0.1 A after (a) $100^{\text {th }}$, (b) 1000 , (c) 5000, and (d) 10000 cycles. 

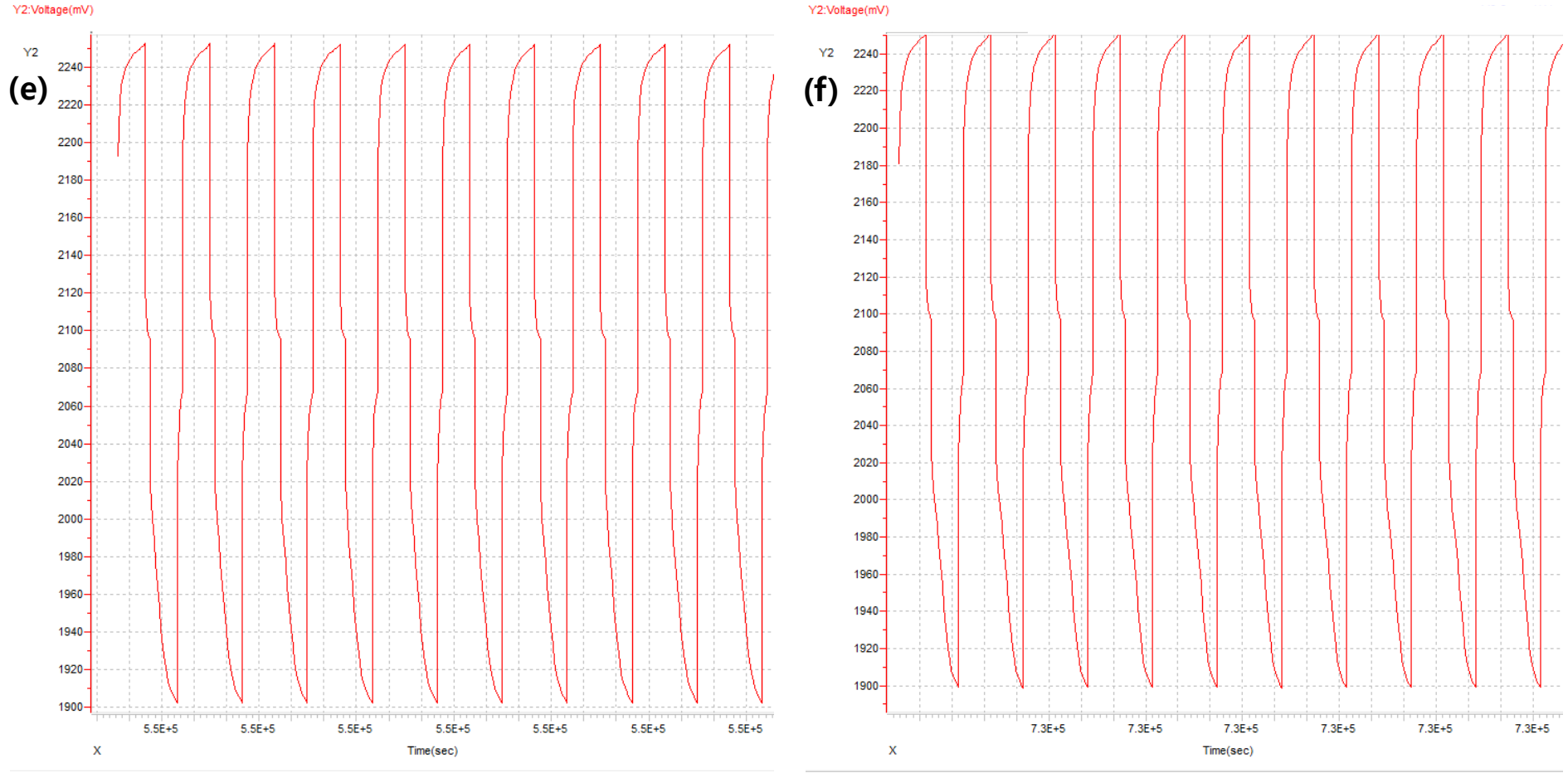

Figure S5. Cycle performance of BC-PE battery at $0.1 \mathrm{~A}$ after (e) 15000 and (f) 20000 cycles. 